\title{
Dependency of Resolvable Gravitational Spatial Resolution on Space-Borne Observation Techniques
}

\author{
P.N.A.M. Visser, E.J.O. Schrama, N. Sneeuw, and M. Weigelt
}

\begin{abstract}
The so-called Colombo-Nyquist (Colombo, The global mapping of gravity with two satellites, 1984) rule in satellite geodesy has been revisited. This rule predicts that for a gravimetric satellite flying in a (near-)polar circular repeat orbit, the maximum resolvable geopotential spherical harmonic degree $\left(l_{\max }\right)$ is equal to half the number of orbital revolutions $\left(n_{\mathrm{r}}\right)$ the satellite completes in one repeat period. This rule has been tested for different observation types, including geoid values at sea level along the satellite ground track, orbit perturbations (radial, along-track, cross-track), low-low satellite-to-satellite tracking, and satellite gravity gradiometry observations (all three diagonal components). Results show that the Colombo-Nyquist must be reformulated. Simulations indicate that the maximum resolvable degree is in fact equal to $k n_{\mathrm{r}}+1$, where $k$ can be equal to 1,2 , or even 3 depending on the combination of observation types. However, the original rule is correct to some extent, considering that the quality of recovered gravity field models is homogeneous as a function of geographical longitude as long as $l_{\max }<n_{\mathrm{r}} / 2$.
\end{abstract}

\subsection{Introduction}

Colombo (1984) has indicated that for exact satellite circular repeat orbits and for continuous space-borne gravimetric observations, the normal matrix of gravity field spherical harmonic ( $\mathrm{SH}$ ) coefficients becomes block-diagonal when organized per $\mathrm{SH}$ order. The

P.N.A.M. Visser $(\bowtie)$ • E.J.O. Schrama

Faculty of Aerospace Engineering, Delft Institute of Earth Observation and Space Systems (DEOS), Delft University of Technology, Kluyverweg 1, 2629 HS, Delft, The Netherlands e-mail: P.N.A.M.Visser@tudelft.nl

N. Sneeuw $\bullet$ M. Weigelt

Institute of Geodesy, University of Stuttgart, GeschwisterScholl-Str. 24D, 70174, Stuttgart, Germany correlation between different orders is zero as long as one can avoid overlapping frequencies, which is generally guaranteed if the maximum resolvable $\mathrm{SH}$ degree $\left(l_{\max }\right)$ is less than half the number of orbital revolutions $n_{\mathrm{r}}$ which the satellite completes in a repeat period of $n_{\mathrm{d}}$ nodal days, or $l_{\max }<n_{\mathrm{r}} / 2$ (Schrama 1990). Although Sneeuw (2000) has pointed out that avoiding overlapping frequencies is fundamentally a restriction on the maximum SH order. Nevertheless this has led to the rule-of-thumb that the maximum resolvable degree is equal to $n_{\mathrm{r}} / 2$, referred to as the Colombo-Nyquist rule. This rule has major implications for the design of future gravity field missions, where several trade-offs have to be made, such as temporal and spatial resolution, the observation/decoupling of different sources of gravity field 
changes, etc. (Bender et al. 2008; Reubelt et al. 2010; Visser and Schrama 2005). Also, this rule has implications for designing efficient gravity field estimation schemes taking advantage of the structure of normal matrices (Schrama 1991). It has to be noted that the maximum resolvable degree is defined as the maximum $\mathrm{SH}$ degree for which also all coefficients with $\mathrm{SH}$ orders complete to this maximum degree can be resolved. It is thus not precluded that certain individual coefficients with a higher $\mathrm{SH}$ degree can be resolved, however with a SH order that is not higher.

The Colombo-Nyquist rule-of-thumb has been tested for a number of mission scenarios, i.e. different repeat orbits and combinations of observables. It is shown that this rule needs to be reformulated. The selected mission scenarios are outlined in Sect. 45.2. The method used for establishing the maximum resolvable degree for these mission scenarios is briefly described in Sect. 45.3. Results are presented in Sect. 45.4 and summarized in Sect. 45.5.

\subsection{Mission Scenarios}

The selected repeat orbits and observable types are listed in Table 45.1. The orbits are polar to ensure global coverage. A repeat orbit is specified by the number of revolutions $n_{\mathrm{r}}$ that is completed in $n_{\mathrm{d}}$ nodal days, where $n_{\mathrm{r}}$ and $n_{\mathrm{d}}$ do not have common prime factors (except 1). Short repeat periods ranging from 1 to 3 days have been selected to limit the computational burden. These short repeat periods are however sufficient to test the validity of the Colombo-Nyquist rule. Different parities for $n_{\mathrm{r}}$ and $n_{\mathrm{d}}$ were selected to assess the possible impact on the maximum resolvable degree of the number of distinct equator crossings. For $n_{\mathrm{r}}-n_{\mathrm{d}}$ even the number of
Table 45.1 Selected polar repeat orbits and observation techniques. The time interval between observations is always taken equal to $1 \mathrm{~s}$

\begin{tabular}{lll}
\hline Repeat period $n_{\mathrm{d}}$ (days) & Number of revolutions $n_{\mathrm{r}}$ & $\begin{array}{l}\text { Height } \\
(\mathrm{km})\end{array}$ \\
\hline 1 & 15 & 554.25 \\
\hline 2 & 31 & 404.35 \\
\hline 3 & 46 & 453.41 \\
\hline Observation technique & 47 & 356.16 \\
\hline Geoid & & Precision level \\
\hline Orbit & & $1 \mathrm{~cm}$ \\
\hline ll-SST & & $1 \mathrm{~cm}$ \\
\hline SGG & & $1 \mu \mathrm{m}$ \\
\hline
\end{tabular}

equator crossings is equal to $n_{\mathrm{r}}$, whereas this is $2 n_{\mathrm{r}}$ for $n_{\mathrm{r}}-n_{\mathrm{d}}$ odd (Fig. 45.1).

The observable types include geoid values at sea level along the satellite ground path (closely related to altimeter observations), orbit perturbations in the radial, along-track and cross-track direction, low-low satellite-to-satellite tracking (ll-SST) range observations, and satellite gravity gradient (SGG) observations (the diagonal components, where the gradiometer instrument is aligned with the radial, alongtrack, and cross-track direction). The observations are assumed to be provided continuously with a constant time step of $1 \mathrm{~s}$. The relation between $\mathrm{SH}$ gravity field coefficients and observations is given by wellestablished and tested transfer functions (e.g. Schrama 1991; Sneeuw 2000; Visser 1992, 2005; Visser et al. 1994, 2001, 2003). These transfer functions are used to set up the observation equations, which are to be solved by the weighted least-squares method (Sect. 45.3). The observations are assigned weights in accordance with the precision levels listed in Table 45.1.
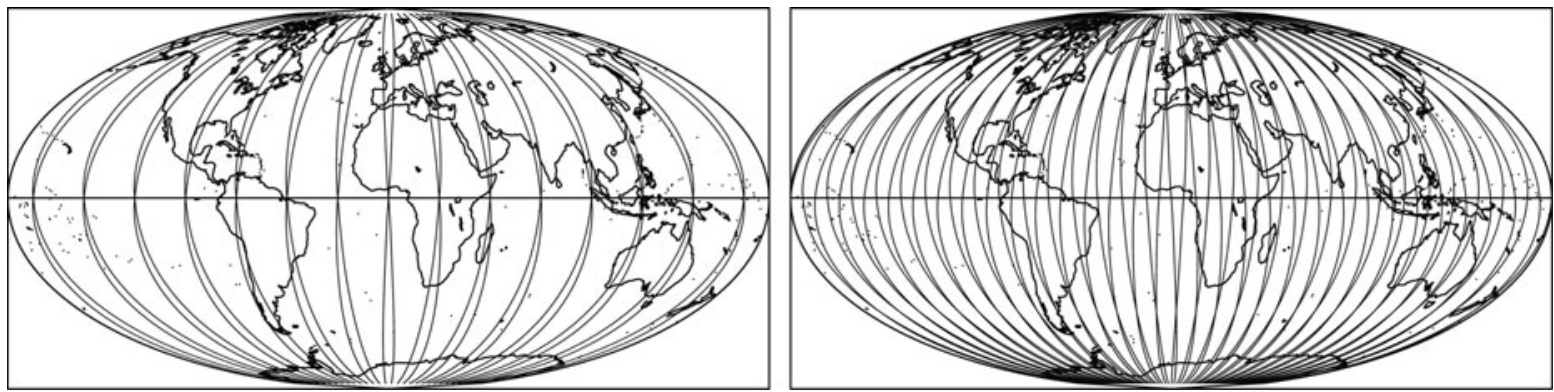

Fig. 45.1 Ground track pattern for polar repeat orbits, where $\mathrm{n}_{\mathrm{r}} / \mathrm{n}_{\mathrm{d}}$ is equal to respectively $15 / 1$ (left) and $31 / 2$ (right) 
Fig. 45.2 Structure of normal matrix for gravity field coefficients complete to degree and order 40 for a $n_{\mathrm{r}} / n_{\mathrm{d}}=15 / 1$ polar repeat orbit based on 11-SST observations ("kite matrix"). Zero values are indicated by white and non-zero by gray color
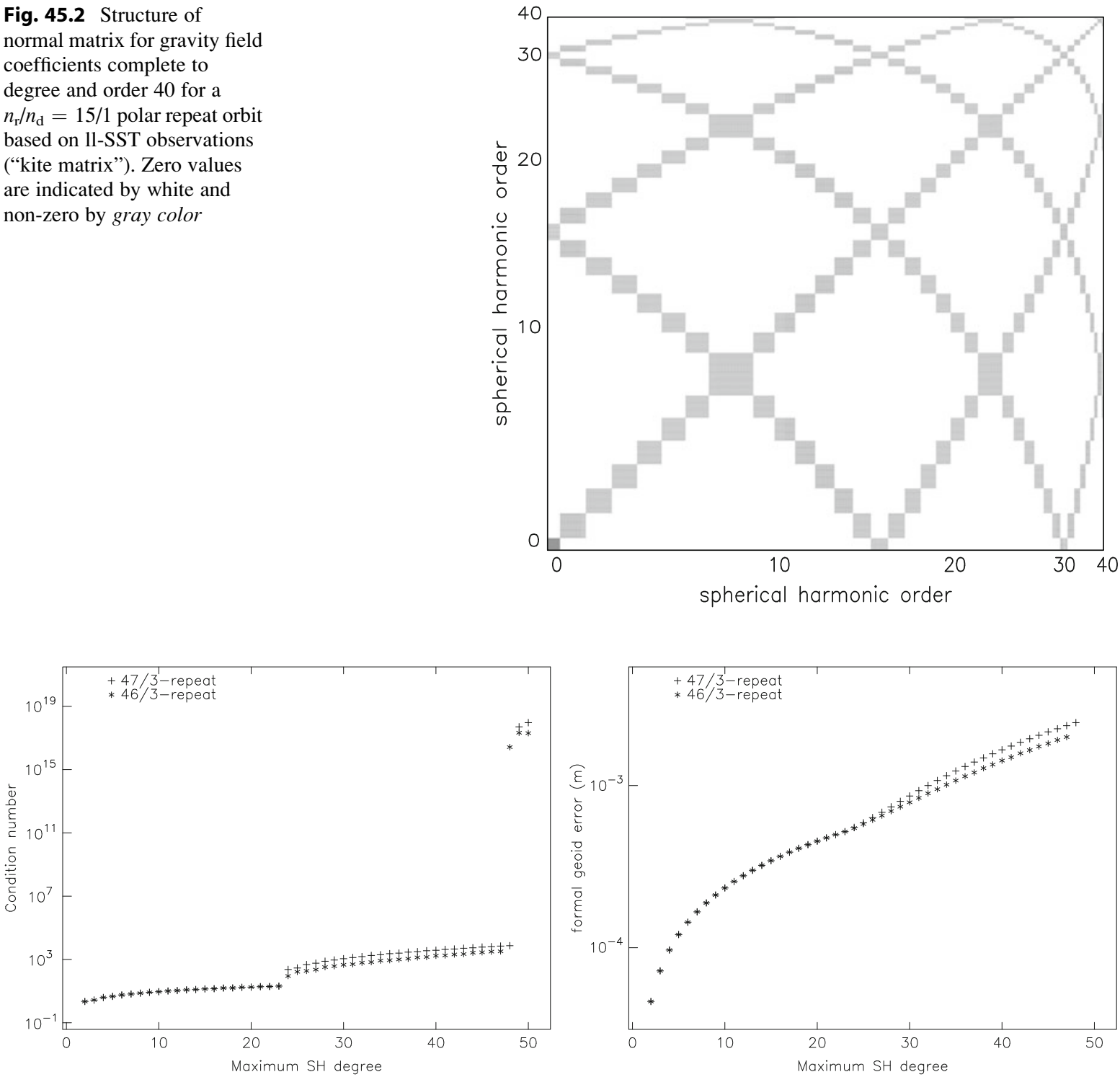

Fig. 45.3 Condition number of the normal equations (left) and global RMS formal geoid error as a function of the maximum retrieved spherical harmonic degree. Use is made of geoid observations at sea level

\subsection{Estimating the Maximum Resolvable Spherical Harmonic Degree}

For a repeat orbit, Colombo (1984) indicated that when a least-squares estimation method is used and if a continuous time series of observations is obtained with constant time interval, the normal matrix for the SH coefficients will become block-diagonal when organized per order, and correlations between different orders will be equal to zero as long as the maximum resolvable degree is below $n_{\mathrm{r}} / 2$. For higher degrees, different orders get correlated and the normal matrix adopts a Kite-like structure (e.g. Fig. 45.2). The question is addressed if still a stable gravity field solution can be obtained in the presence of these correlations, thereby assuming that no use is made of prior knowledge and/or regularization. This is tested by computing the condition number of this matrix 

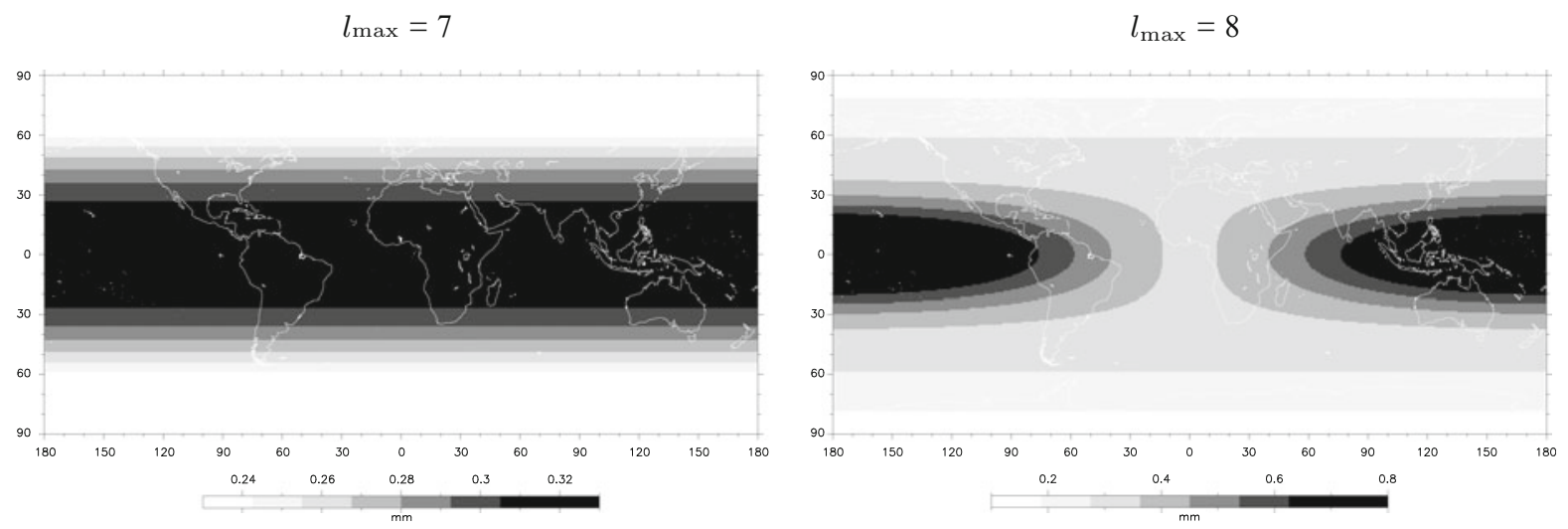

Fig. 45.4 Formal geoid error as a function of the geographical location for geoid observations along a $n_{\mathrm{r}} / n_{\mathrm{d}}=15 / 1$ repeat orbit

Table 45.2 Formal global geoid error (mm) (RMS, minimum and maximum) and the ratio of maximum and minimum geoid error at the equator $\left(\rho_{\text {eq }}\right)$ for $n_{\mathrm{r}} / n_{\mathrm{d}}=15 / 1$ and $31 / 2$ repeat orbits. For $l_{\max }<n_{\mathrm{r}} / 2$ the error is always constant as a function of longitude

\begin{tabular}{|c|c|c|c|c|c|}
\hline Obs. & $l_{\max }$ & RMS & $\rho_{\mathrm{eq}}$ & Minimum & Maximum \\
\hline \multicolumn{6}{|c|}{$n_{\mathrm{r}} / n_{\mathrm{d}}=15 / 1$-repeat } \\
\hline Geoid & 7 & 0.2632 & 1.00 & 0.1333 & 0.3200 \\
\hline Geoid & 8 & 0.3976 & 1.08 & 0.1419 & 0.9105 \\
\hline Geoid & 15 & 1.7342 & 12.54 & 0.1924 & 6.4213 \\
\hline $11-S S T$ & 7 & 0.0023 & 1.00 & 0.0013 & 0.0028 \\
\hline 11-SST & 8 & 0.0027 & 1.00 & 0.0014 & 0.0034 \\
\hline $11-S S T$ & 15 & 0.0076 & 1.37 & 0.0059 & 0.0109 \\
\hline \multicolumn{6}{|c|}{$n_{\mathrm{r}} / n_{\mathrm{d}}=31 / 2$-repeat } \\
\hline Geoid & 15 & 0.3810 & 1.00 & 0.1350 & 0.4724 \\
\hline Geoid & 16 & 0.4092 & 1.00 & 0.1392 & 0.5314 \\
\hline Geoid & 32 & 1.4984 & 1.00 & 0.1922 & 4.9795 \\
\hline $11-S S T$ & 15 & 0.0033 & 1.00 & 0.0013 & 0.0038 \\
\hline $11-\mathrm{SST}$ & 16 & 0.0036 & 1.00 & 0.0014 & 0.0042 \\
\hline $11-S S T$ & 31 & 0.0108 & 1.00 & 0.0041 & 0.0139 \\
\hline
\end{tabular}

(ratio of maximum and minimum eigenvalue) and by computing the Root-Mean-Square (RMS) of the cumulative global formal geoid commission error for the estimated SH coefficients. The formal geoid errors were taken from the inverse (if the normal matrix is invertible) of the weighted normal matrix. In all cases, normal equations were set up for all SH coefficients from degree 2 to a certain maximum degree $l_{\max }$. Thus the impact of omission and/or aliasing of unmodeled gravity field sources are not taken into account. The exercises described in this paper only address the issue of observability of a static gravity field complete to the maximum $\mathrm{SH}$ degree solved for.

\subsection{Gravity Field Observability}

As a first test case, the condition numbers of the normal matrix and associated geoid error were computed for $n_{\mathrm{r}} / n_{\mathrm{d}}=46 / 3$ and $n_{\mathrm{r}} / n_{\mathrm{d}}=47 / 3$ repeat orbits using geoid observations along the ground track. The condition numbers display a large jump at $l_{\max }=n_{\mathrm{r}}$ (Fig. 45.3, left) and in fact the normal matrix could not be inverted for higher degrees (no formal geoid errors could be estimated, Fig. 45.3, right). For $l_{\max }=n_{\mathrm{r}} / 2$, a small jump in the condition number occurs due to the additional correlations between different SH orders, but this does not lead to an unstable normal matrix. Also, the slope of the geoid error increases for $l_{\max }>n_{\mathrm{r}} / 2$. Based on these results, it can already be concluded that the maximum resolvable degree can be as big as $n_{\mathrm{r}}$ and does not depend on the parity of $n_{\mathrm{r}}$ and $n_{\mathrm{d}}$.

It is interesting to note that as long as $l_{\max }<n_{\mathrm{r}} / 2$, the geoid error is only latitude dependent and does not change with longitude, whereas for $l_{\max }>n_{\mathrm{r}} / 2$ the correlations between different orders cause the geoid error to change as a function of longitude as well (Fig. 45.4). The variation of the geoid error as a function of latitude and longitude depends on the observable. For a $n_{r} / n_{d}=15 / 1$ repeat orbit and geoid observations, the minimum and maximum formal geoid error is equal to 0.19 and $6.42 \mathrm{~mm}$ for a gravity field recovery complete to degree and order 15 , i.e. a ratio of 34, compared 0.0059 and 0.0109 or a ratio of 1.8 for ll-SST observations (Table 45.2).

Figures 45.5 and 45.6 display the condition numbers of the normal matrix and formal geoid error 
$n_{\mathrm{r}} / n_{\mathrm{d}}=15 / 1$ polar repeat orbit
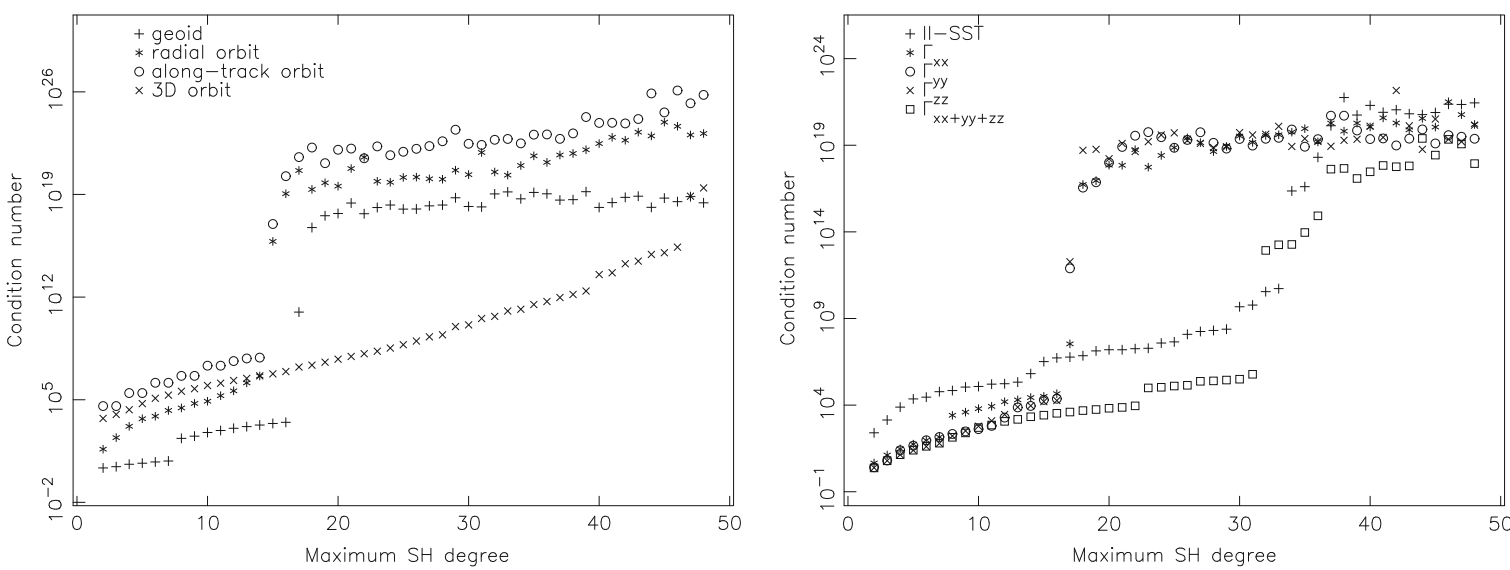

$n_{\mathrm{r}} / n_{\mathrm{d}}=31 / 2$ polar repeat orbit
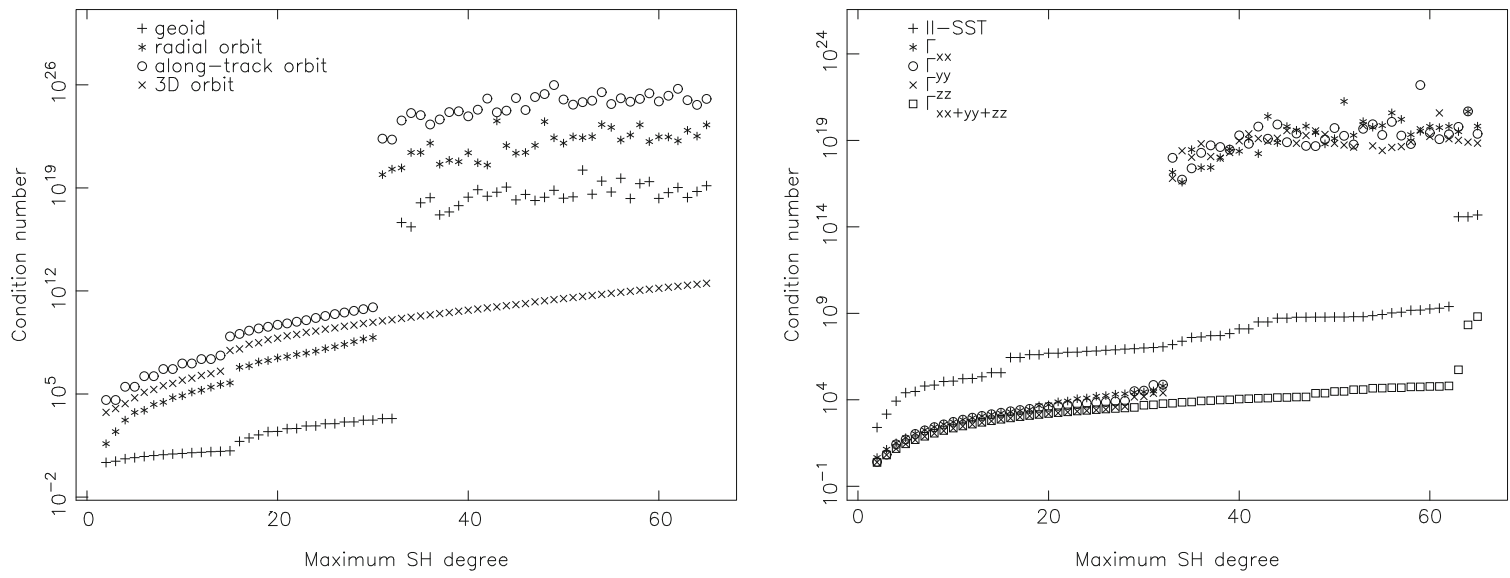

Fig. 45.5 Condition number of the normal equations as a function of the maximum retrieved spherical harmonic degree and the observation technique (the minimum degree is equal to 2 )

estimates for gravity field recoveries up to $l_{\max }=50$ for the $n_{\mathrm{r}} / n_{\mathrm{d}}=15 / 1$ repeat orbit, i.e. $l_{\max }>3 n_{\mathrm{r}}+1$, and up to $l_{\max }=65$ for the $n_{\mathrm{r}} / n_{\mathrm{d}}=31 / 2$ repeat period, i.e. $l_{\max }>2 n_{\mathrm{r}}+1$. The observable types include (1) geoid values, (2) radial orbit perturbations, (3) alongtrack orbit perturbations, (4) orbit perturbations in all directions (3D), (5) along-track diagonal gravity gradient component $\left(\Gamma_{\mathrm{xx}}\right)$, (6) cross-track diagonal gravity gradient component $\left(\Gamma_{\mathrm{yy}}\right)$, (7) radial diagonal gravity gradient component $\left(\Gamma_{\mathrm{xx}}\right)$, and (8) all three diagonal gravity gradient components $\left(\Gamma_{\mathrm{xx}+\mathrm{yy}+\mathrm{zz}}\right)$.

It can be observed that for one-directional observables, such as geoid values, radial orbit perturbations, along-track perturbations, and one diagonal of the gravity gradients, the condition numbers display in general small jumps at $n_{\mathrm{r}} / 2$ and large jumps at $n_{\mathrm{r}}+1$. The same can be observed for the associated geoid error estimate (provided the normal matrix was invertible). In other words, for such one-directional observables it seems like the maximum resolvable $\mathrm{SH}$ degree is equal to the number of revolutions $n_{\mathrm{r}}+1$ in a repeat period. When using 11-SST observations, combinations of orbit perturbations (3D) or combinations of SGG diagonal components, the normal matrix is stable up to at least $l_{\max }=2 n_{\mathrm{r}}+1$. For the $3 \mathrm{D}$ combination of orbit perturbations, the condition number and associated formal geoid error estimate stays stable for $l_{\max }+1$ up to $3 n_{\mathrm{r}}+1$, whereas for the combination of all three diagonal SGG components, this is still $2 n_{\mathrm{r}}+1$.

Two questions that might now immediately be raised is why this is not $3 n_{\mathrm{r}}+1$ for the combination 
$n_{\mathrm{r}} / n_{\mathrm{d}}=15 / 1$ polar repeat orbit
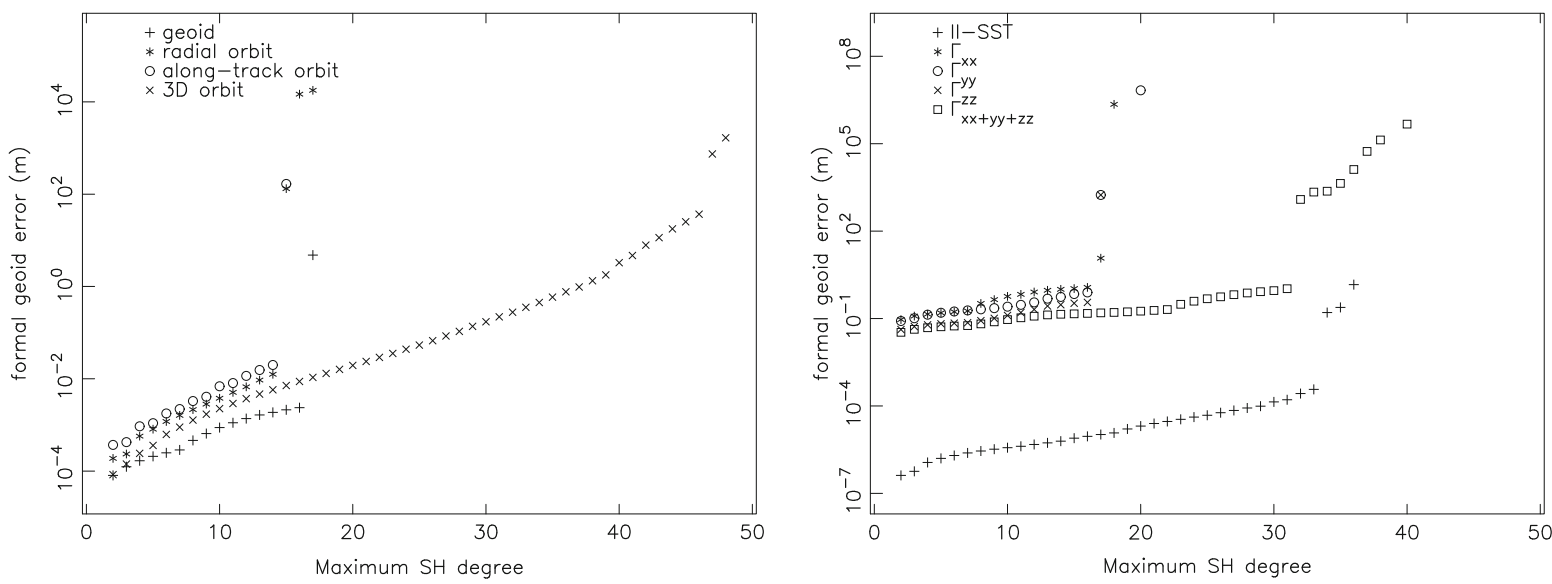

$n_{\mathrm{r}} / n_{\mathrm{d}}=31 / 2$ polar repeat orbit
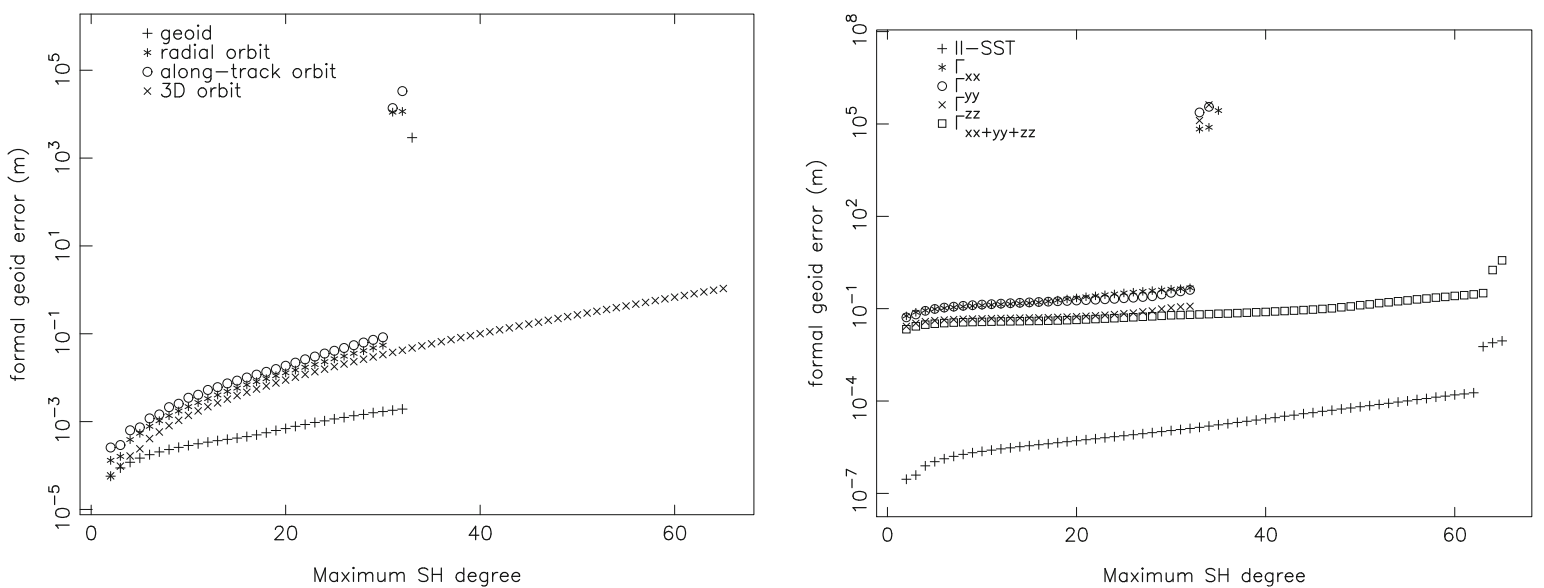

Fig. 45.6 Global RMS formal geoid error from the inverse of the normal equations as a function of the maximum retrieved spherical harmonic degree and the observation technique (the minimum degree is equal to 2 )

of three SGG components as well and why it is $2 n_{\mathrm{r}}+1$ for ll-SST observations, which is a one-directional observation type, namely along the line-of-sight between two trailing satellites. Concerning the SGG observations, it can be argued that the three diagonal components are not independent because the gravitational potential satisfies the Laplace equation, or $\Gamma_{\mathrm{xx}}+$ $\Gamma_{\mathrm{yy}}+\Gamma_{\mathrm{zz}}=0$. Thus one diagonal SGG components can always be written as a linear combination of the other two. Thus, in fact only two independent components remain. Concerning the 11-SST observations, it can be argued that these observations are a modulated combination of along-track and radial orbit perturbations (Visser 2005), assuming the two associated satellites fly in the same orbital plane.

\section{Conclusions}

Computations have shown that the Colombo-Nyquist rule in satellite geodesy, which predicts that the maximum resolvable degree is equal to half the number of orbital revolutions $n_{\mathrm{r}}$ in a repeat period of $n_{\mathrm{d}}$ nodal days, requires revision. Colombo's rule is correct in the sense that block-diagonal matrices are formed when $l_{\max }<$ $n_{\mathrm{r}} / 2$ and when organized per $\mathrm{SH}$ order, with no correlations between the orders. Colombo's rule is in general too pessimistic to infer statistical significance of SH coefficients in a gravity field model, i.e. solutions are possible where $l_{\max } \geq n_{\mathrm{r}} / 2$ as is discussed in this paper. If the maximum degree of 
estimated SH coefficients is larger than $n_{\mathrm{r}} / 2$, the gravity field solution will however no longer be homogeneous in the longitude direction for even parities of $n_{\mathrm{r}}$ and $n_{\mathrm{d}}$. However, the Colombo-Nyqyist rule can be considered to be correct to some extent. Namely, as stated in the previous paragraph, the quality of recovered gravity field models is always homogeneous as a function of geographical longitude as long as $l_{\max }<n_{\mathrm{r}} / 2$.

It was also found that the maximum resolvable degree does not depend on the parity of the number of revolutions and nodal days in a repeat orbit, but that the recovery error as a function of longitude does vary due to the increasing ground track density when traveling away from the equator. Finally, the maximum resolvable degree depends on the (combination of) observable type(s). In case of combinations of independent observables, this maximum degree can be one, two or three times the number of orbital revolutions in a repeat period (plus 1 if the minimum $\mathrm{SH}$ degree is taken equal to 2). Fortunately, in general gravity satellites carry a complement of observing instruments, including always GPS receivers in addition to for example 11-SST instruments or a gradiometer.

\section{References}

Bender PL, Wiese DN, Nerem RS (2008) A possible dualGRACE mission with 90 degree and 63 degree inclination orbits. In: ESA (ed) 3rd International symposium on formation flying, missions and technologies, 23-25 April 2008, ESA/ESTEC, Noordwijk, pp 1-6
Colombo OL (1984) The global mapping of gravity with two satellites, vol 7, no 3, Publications on geodesy, New Series. Netherlands Geodetic Commission, Delft

Reubelt T, Sneeuw N, Sharifi MA (2010) Future Mission Design Options for Spatio-Temporal Geopotential Recovery. International Association of Geodesy Symposia. Vol. 135, Springer, pp 163-170

Schrama EJO (1990) Gravity field error analysis: applications of GPS receivers and gradiometers on low orbiting platforms, NASA technical memorandum 100679. GSFC, Greenbelt, MD

Schrama EJO (1991) Gravity field error analysis: applications of GPS receivers and gradiometers on low orbiting platforms. J Geophys Res 96(B12):20041-20051

Sneeuw N (2000) A semi-analytical approach to gravity field analysis from satellite observations. $\mathrm{PhD}$ dissertation, Deutsche Geodätische Kommission, Reihe C, Nr. 527

Visser PNAM (1992) The use of satellites in gravity field determination and model adjustment. PhD dissertation, Delft University of Technology, Delft

Visser PNAM (2005) Low-low satellite-to-satellite tracking: applicability of analytical linear orbit perturbation theory. J Geod 79(1-3):160-166

Visser PNAM, Schrama EJO (2005) Space-borne gravimetry: how to decouple the different gravity field constituents? In: Jekeli $\mathrm{C}$ et al (eds) Gravity, geoid and space missions, vol 129, International association of geodesy symposia. Springer, Berlin, pp 6-11

Visser PNAM, Wakker KF, Ambrosius BAC (1994) Global gravity field recovery from the ARISTOTELES satellite mission. J Geophys Res 99(B2):2841-2851

Visser PNAM, van den IJssel J, Koop R, Klees R (2001) Exploring gravity field determination from orbit perturbations of the European Gravity Mission GOCE. J Geod 75(2/3):89-98

Visser PNAM, Sneeuw N, Gerlach C (2003) Energy integral method for gravity field determination from satellite orbit coordinates. J Geod 77(3/4):207-216 